\title{
Changes in subject performance during the semester: An empirical investigation
}

\author{
WILLIAM LANGSTON, CLARK OHNESORGE, PETER KRULEY, and STEVEN J. HAASE \\ University of Wisconsin, Madison, Wisconsin
}

\begin{abstract}
Two experiments were conducted to probe for performance differences between early-semester subjects and late-semester subjects in an introductory psychology subject pool. In Experiment 1A, hypotheses regarding changes in performance included differential effort, changes in subjects' attention to the task, and changes in subjects' sensitivity. A signal detection paradigm was used, and 278 subjects were sampled over the course of the semester. No evidence of changes in subject performance was found, in spite of sufficient power to detect relevant patterns of change. The results of a replication $(N=135)$ were consistent with those of Experiment $1 \mathrm{~A}$. Experiment 2 $(N=118)$ employed a text comprehension task to examine possible differences in subject performance with a more cognitive task. Again, there was no evidence to support the idea that latesemester subjects were performing differently from early-semester subjects.
\end{abstract}

In the field of psychology, details of experimental design are thoroughly scrutinized. Particularly when the experimental task is complex, great care must be taken to ensure that the subject is attempting to follow the directions of the experimenter. A foundational assumption for any experiment not explicitly investigating subject differences is that the population of subjects sampled is homogeneous. Systematic changes in subject behavior across the semester could influence the pattern of results or reduce the power of an experimental manipulation. Additionally, if systematically different groups are sampled, the generalizability of the conclusions may be seriously reduced.

Interestingly, the belief is common among researchers utilizing an undergraduate subject pool that students $d o$ vary in important respects across the semester. Subjects participating in the early weeks of the semester are usually believed to follow directions more closely or attend more fully during the experiment.

Several studies have been conducted yielding significant differences between early-semester subjects and latesemester subjects on such demographic attributes as academic orientation and locus of control (Evans \& Donnerstein, 1974), preoccupation with death (Blatt \& Quinlan, 1967), and respect for the scientific enterprise (Holmes, 1967). However, absent theoretical connections between such indices and performance for a given task, these re-

\footnotetext{
The authors would like to thank Arthur Glenberg, Yei-Yu Yeh, Mike Hogan, Steve Morgan, and Bob Kachelski for their helpful comments on an earlier version of the manuscript. We thank John Theios and Arthur Glenberg for the use of their lab space and equipment in conducting the experiment. The order of the first and second and the third and fourth authors was determined by a coin flip. Peter Kruley was partially supported by AASERT Grant F49620-92-J-0310 from the Air Force Office of Sponsored Research during preparation of this report. Requests for reprints may be sent to William Langston, Department of Psychology, 1202 W. Johnson, Madison, WI 53706.
}

sults provide little support for the inference that earlysemester subjects generally produce higher quality data.

Changes in performance across the semester have also been measured. Underwood, Schwenn, and Keppel (1964) found no support for a decline in subject performance across the semester on a simple paired-associate learning task. In comparisons of performance on the Wechsler Adult Intelligence Scale (WAIS), Blatt and Quinlan (1967) found no difference for the information and vocabulary subtests, but did find a significant advantage for earlysemester subjects on the picture arrangement subtest. Richter, Wilson, Milner, and Senter (1981) compared early-semester and late-semester subjects' performance on two tasks, serial learning and symbol substitution. Latesemester subjects performed significantly better on the serial learning task. However, early-semester subjects surpassed their counterparts in both mean number attempted and percent correct on the symbol substitution task. Given the lack of consensus among these studies, the possibility of real differences in subject performance across the semester remains an open question.

Our goal is to probe for subject differences based on the following possibilities: (1) Early subjects are somehow just better (2) Late subjects employ less conservative response strategies (3) Early subjects are more conscientious in their attention to directions and try harder (4) Late subjects are less consistent (the population is more heterogeneous).

\section{EXPERIMENT 1A}

The hypotheses above were tested using a methodology based on the theory of signal detectability (Green \& Swets, 1966). Signal detection methodology allows for assessments of sensitivity $\left(d^{\prime}\right)$, a subject's ability to make correct classifications, and bias $(\beta)$, a subject's willingness to make a particular classification. In addition, reac- 
tion times can be collected and used to draw inferences about subjects' effort.

The hypothesis that early subjects are better can be viewed as a prediction that subject sensitivity will decline as the semester progresses. The two most relevant possible patterns of change in $d^{\prime}$ are (1) $d^{\prime}$ remains stable over the first 10-12 weeks and then drops off sharply in the final weeks (possibly due to stress from final exams) or (2) $d^{\prime}$ decreases linearly across the semester (because "poorer" subjects participate later).

If late subjects are less conservative, one would expect changes in bias. These changes could arise for a number of reasons. For example, subjects who participate later in the semester might be more tired, leading them to adopt a more extreme response criterion to decrease the burden imposed by the experiment, or some uncooperative late subjects might just press one response key for all trials.

If early subjects try harder, one would expect changes in reaction times. In particular, early subjects should respond faster, on average, than later subjects. Evidence for the effort hypothesis might also be found in the interaction between block of trials in the experiment and week of the semester. For example, subjects at the beginning of the semester should show more of a speed-up in reaction time across blocks than should subjects at the end of the semester.

The heterogeneity hypothesis predicts that the proportion of "poor" subjects increases as the semester progresses. So, at the end of the semester, the modal subject will be just as conscientious as the modal subject at the beginning of the semester, but the probability that some individual subject will respond in an aberrant fashion will increase. If this hypothesis is correct, one would expect the variability of responses to be greater at the end of the semester than at the beginning.

\footnotetext{
Method

Subjects. Two hundred seventy-eight right-handed students in the University of Wisconsin-Madison subject pool participated in this experiment in exchange for extra credit. On average, 20 subjects participated in each of the 14 weeks in the spring semester of 1993.

Design. The design was a mixed factorial: weeks was the between factor with 14 levels, and block of trials in the experiment was the within factor with 7 levels. The three dependent measures were $d^{\prime}, \beta$, and reaction time.

Procedure. Data were collected using Macintosh II and Macintosh Classic computers. The subjects read the instructions from the computer screen at their own pace. They were told that they would see a number of screens containing an array of " $\mathrm{C}$ "s and that on half of the screens a " $G$ " would also be present. The subjects were instructed to respond "yes" or "no" to the question, "Was there a G in the display you just saw?" The subjects used the computer keyboard to respond; "yes" was indicated with the dominant hand.

The subjects began with a practice block of trials that was the same as the experimental blocks. Each block contained 42 trials of the signal detection task. There were 20 characters on the screen for each trialeither 20 "C" $\mathrm{C}$ "s or 19 " C"'s and one "G." Screen locations for the 20 characters were chosen at random for each trial. The font for each character was 12-point bold Geneva.

Each display was presented for approximately $117 \mathrm{msec}$. The timer started as soon as the last character was drawn. After each display, the screen was erased and the prompt "Response?" appeared. This prompt remained on the screen until the subject responded. The subjects then
}

received feedback about whether or not they were correct on the trial. The feedback was on the screen for approximately $417 \mathrm{msec}$, and then a new trial began.

The experimental session was composed of seven blocks of trials in addition to the practice block, and the subjects were allowed to rest between blocks. The entire session took approximately $30 \mathrm{~min}$ to complete.

The debriefing described the experiment in terms of visual perception and did not focus on the investigation of changes in subject performance. This was done to avoid possible contamination of subjects to be run later in the semester.

\section{Results}

Presentation of the results is organized around tests of the four hypotheses discussed in the introduction. Data from the practice block were not included. The probability of a Type I error for all statistical tests was set at .05 . Also, except where noted, the analyses were conducted on data aggregated across blocks of trials.

Hypothesis 1. Subjects' sensitivity decreases over the course of the semester. Each subject's overall $d^{\prime}$ was computed. These data, illustrated in the top left panel of Figure 1, were then analyzed in a one-way betweensubjects analysis of variance (ANOVA), with week of the semester as the independent variable. Because this omnibus $F$ test of a source with $13 d f \mathrm{~s}$ may not be the most powerful test of specific hypothesized changes in performance, two additional analyses were conducted as planned comparisons: (1) a simple linear regression on the overall $d^{\prime}$ scores using week of semester as a predictor, corresponding to a pattern of steady decline across the semester, and (2) a single- $d f$ contrast, also computed as a simple regression, in which scores from the first 12 weeks of the semester were compared with those in Weeks 13 and 14, testing whether late-semester subjects performed more poorly than other subjects. Table 1 shows the $F$ ratios obtained in these analyses, along with estimates of the observed effect size, $\omega^{2}$ (see Keppel, 1991, p. 439 , for the method used to estimate $\omega^{2}$ ). All hypothesis tests were nonsignificant; furthermore, the estimated $\omega^{2}$ s were all less than .005-that is, less than one half the size of Cohen's (1988) canonical small effect. Thus, there was no support for the hypothesis that early subjects' sensitivity is different from late subjects' sensitivity.

Hypothesis 2. Late subjects employ less conservative response strategies. Three analyses similar to the ones above were performed using $\beta$ as the dependent measure. The mean values of $\beta$ for each week of the semester are illustrated in the middle left panel of Figure 1. These analyses (in Table 1) all yielded nonsignificant results and estimated $\omega^{2}$ s less than .005 . There was no evidence for the hypothesis that early subjects have different response strategies than late subjects.

Hypothesis 3. Early subjects try harder or work more diligently at the task. The median ${ }^{1}$ reaction time for correct trials was computed for each subject. The means for the median reaction times for each week are illustrated in the bottom left panel of Figure 1. These data were analyzed in the same way as were the $d^{\prime}$ and $\beta$ dependent measures. In these analyses, shown in Table 1, all outcomes were nonsignificant, and all estimated $\omega^{2} \mathrm{~s}$ were less than .005 . 

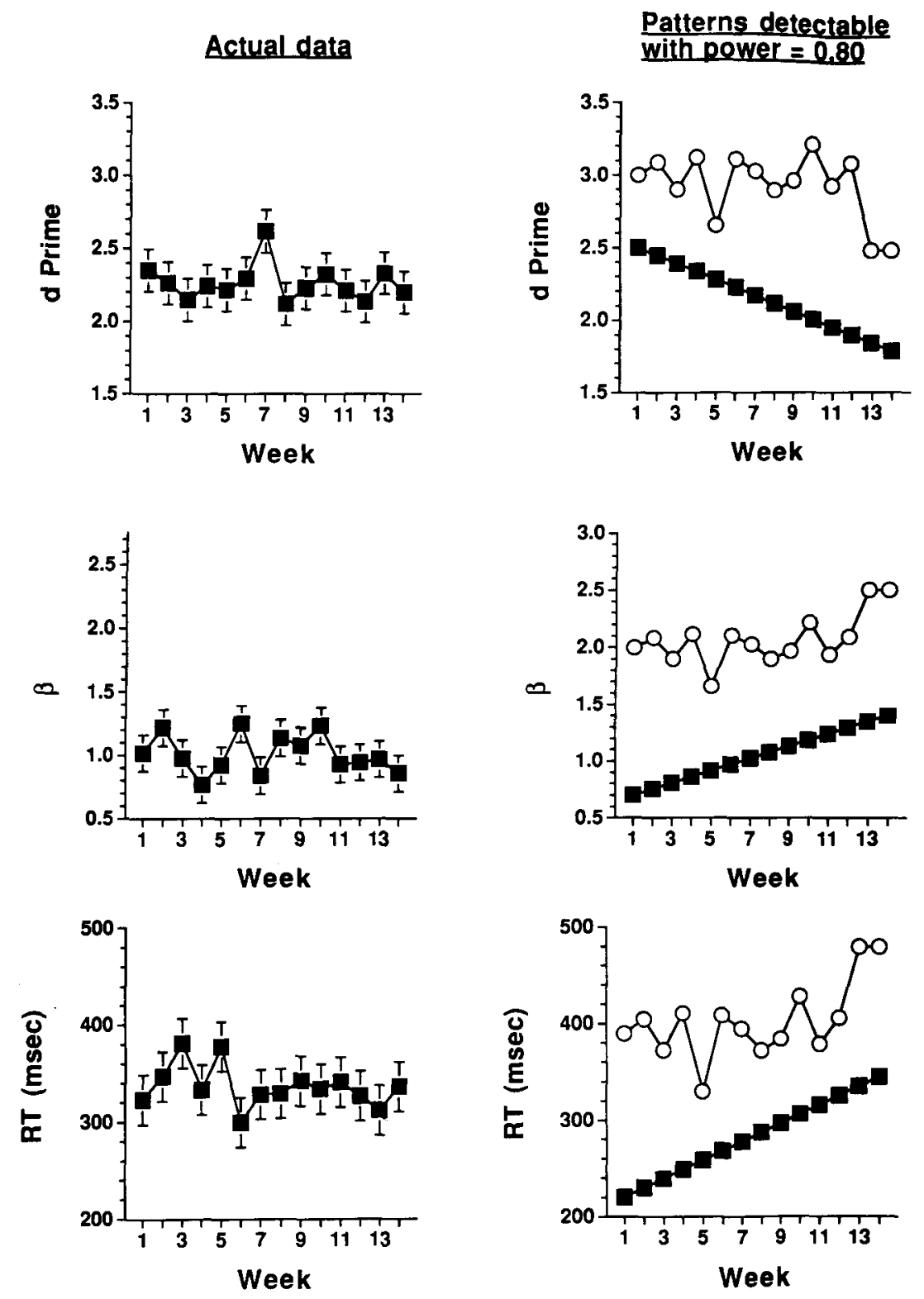

Figure 1. Weekly mean scores on the variables $d^{\prime}$, bias $(\beta)$, and reaction time in Experiment $1 \mathrm{~A}$ are shown in the left-hand panels; error bars extend one standard error above and below the mean, using the ANOVA $M S_{\mathrm{e}}$ as an estimate of population variance. The righthand panels show hypothetical outcomes that could have been detected with a probability of .80; see text for detailed discussion.

The median reaction time was also computed for each of the seven blocks of trials for each subject. These data were then analyzed in a two-way mixed ANOVA, with week of semester as the between-subjects factor and block of trials as the within-subjects factor. Although the main effect for week (which had been analyzed in the one-way ANOVA above) was not significant in this analysis, there was a significant main effect for block of trials $[F(6,1584)$ $\left.=76.99, M S_{\mathrm{e}}=4,625.21\right]$. The subjects showed a steady decrease in reaction time over the course of the experiment. Most importantly, the interaction between block of trials and week was not significant $[F(78,1584)=0.82$,
$M S_{\mathrm{e}}=4,625.21$, estimated $\left.\omega^{2}=.00\right]$. The fact that early-semester subjects and late-semester subjects showed the same amount of improvement across blocks is strong evidence against the differential effort hypothesis.

Hypothesis 4. Early subjects are more consistent: The subject pool is more homogeneous early in the semester. To test this hypothesis, the Brown-Forsythe test of homogeneity of variance (Keppel, 1991) was performed on the three dependent measures obtained in the experiment; for each dependent variable, the absolute deviation of each score from the median score for the week in which it was collected was computed. These absolute deviations 
Table 1

Significance Tests Performed in Experiments 1A and 1B

\begin{tabular}{|c|c|c|c|c|c|}
\hline & $d f$ & $F_{\text {Critical }}$ & $F_{\text {Obtained }}$ & $M S_{\mathrm{e}}$ & Est. $\omega^{2}$ \\
\hline \multicolumn{6}{|c|}{ Analyses of Raw Data } \\
\hline$d^{\prime}$ & & & & & \\
\hline 13-level ANOVA & $(13,264)$ & 1.75 & 0.61 & 0.43 & .00 \\
\hline Linear regression & $(1,276)$ & 3.88 & 0.10 & 0.42 & .00 \\
\hline Contrast & $(1,276)$ & 3.88 & 0.01 & 0.42 & .00 \\
\hline Experiment 1B & $(2,132)$ & 3.06 & 1.39 & 0.41 & .01 \\
\hline \multicolumn{6}{|l|}{1} \\
\hline 13-level ANOVA & $(13,264)$ & 1.75 & 1.05 & 0.41 & .00 \\
\hline Linear regression & $(1,276)$ & 3.88 & 0.62 & 0.41 & .00 \\
\hline Contrast & $(1,276)$ & 3.88 & 1.36 & 0.41 & .00 \\
\hline Experiment 1B & $(2,132)$ & 3.06 & 1.47 & 0.28 & .01 \\
\hline \multicolumn{6}{|l|}{ Reaction Time } \\
\hline 13-level ANOVA & $(13,264)$ & 1.75 & 0.76 & $13,025.25$ & .00 \\
\hline Linear regression & $(1,276)$ & 3.88 & 1.10 & $12,874.83$ & .00 \\
\hline Contrast & $(1,276)$ & 3.88 & 0.74 & $12,891.18$ & .00 \\
\hline Experiment 1B & $(2,132)$ & 3.06 & $3.13^{*}$ & $11,071.86$ & .03 \\
\hline
\end{tabular}

*Significant at .05 level.

were used as data for the same three analyses that were performed on the raw data scores: a one-way ANOVA with 13 levels of week of semester, a linear regression on week of semester, and a contrast comparing Weeks 13 and 14 with the remainder of the semester. These analyses were all nonsignificant, and all yielded estimated $\omega^{2} s$ less than .005. Thus, no evidence was found for changes in the variability of any of these measures across the semester.

Power analyses. Most of the principal conclusions drawn from this experiment are based on null results; accordingly, it is instructive to consider the level of statistical power available in this study. We present power analyses for four significance tests: those made on the dependent variables of $d^{\prime}, \beta$, and reaction time in one-way ANOVAs, and the test of the block of trials $X$ week interaction in the twoway analysis. For each of these tests, the effect size necessary to produce a power of .80 was calculated. Because the $F$ tests in the one-way ANOVAs were computed on the same number of degrees of freedom, the power of these tests was identical: an effect size, $f$, of .27 could have been detected with power of $.80\left(f=s_{\mathrm{m}} / s_{\mathrm{e}}\right.$; see Cohen, 1988 , p. 275) $;^{2}$ this corresponds to an $\omega^{2}$ of .07 . Thus, the tests conducted in these analyses had good power to detect a medium-sized effect, according to Cohen's criteria. For the test of the block of trials $\times$ week interaction, an $f$ of $.24\left(\omega^{2}\right.$ of .05$)$ could have been detected with power greater than .80 .

The magnitude of an effect of the requisite size is shown graphically in the right-hand panels of Figure 1: for each dependent variable, two possible outcomes corresponding to an $f$ of .27 were produced, using the ANOVA $M S_{\mathrm{e}}$ as an estimate of the population variance. One of these outcomes (the filled squares in Figure 1) is a steady decline in performance across the semester. The other (open circles in Figure 1) represents constant subject performance over the first 12 weeks followed by a decline during the last 2 weeks of the semester; this pattern was obtained as follows: the first 12 values were drawn randomly from a normal distribution with variance equal to $M S_{\mathrm{e}} / n$ (the average weekly value of 20 subjects was used for $n$ ), and the points for Weeks 13 and 14 were chosen to produce an overall $f$ of .27 . Note that the pattern across weeks (i.e., the size of the intervals between data points) is relevant for the power analysis. The two patterns in the right panels of Figure 1 were assigned different absolute locations merely for convenience in graphing the data.

\section{Discussion}

The results of the present experiment fail to support any of the four hypotheses relating time of subject participation to the performance indices of $d^{\prime}, \beta$, and reaction time. Because the conclusions were based upon null results, however, we performed a replication of the experiment in the following semester.

\section{EXPERIMENT 1B}

\section{Method}

Subjects, Design, and Procedure. The subjects were right-handed students volunteering for extra credit; 135 students contributed data, which were collected three times during the semester: early (Weeks 1 and 2), middle (Weeks 7 and 8), and late (Weeks 13 and 14). Thus, the experiment was run as a mixed factorial design, with time of semester as the between factor with three levels, and block of trials as the within factor with seven levels. The procedure for the experimental trials was the same as in Experiment 1A.

\section{Results}

The variables $d^{\prime}$ and $\beta$ were analyzed in a one-way between-subjects ANOVA; the results were as shown in Table 1. As in Experiment 1A, the results of $F$ tests were nonsignificant. Estimated $\omega^{2}$ s were less than .015. Reaction time was analyzed in a two-way mixed ANOVA as described above; mean reaction times for each combination of block and time of semester are shown in Figure 2. In this analysis, the block $\times$ time of semester interaction was significant $\left[F(12,792)=2.47, M S_{\mathrm{e}}=3,526.22\right.$, estimated $\left.\omega^{2}=.02\right]$. The main effect of blocks was also significant $\left[F(6,792)=36.77, M S_{\mathrm{e}}=3,526.22\right]$, as was the main effect of time of semester (see Table 1). 


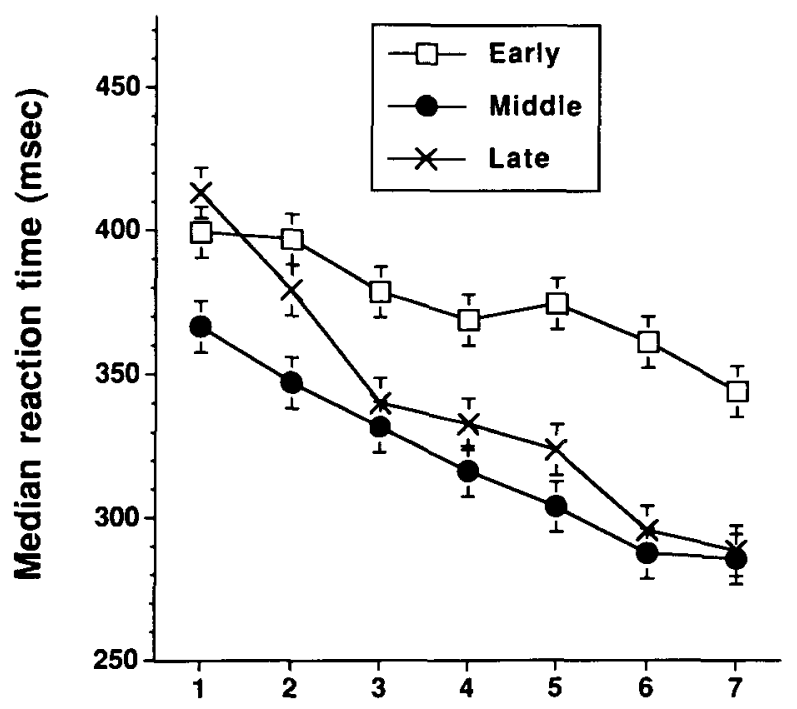

Block of trials

Figure 2. Means of subjects' median reaction times in Experiment $1 B$ for each block of trials for each time of the semester. $\mathrm{Er}$ ror bars extend one standard error above and below the mean, using the ANOVA $M S_{e}$ as a variance estimate.

\section{Discussion}

With respect to the $d^{\prime}$ and $\beta$ dependent measures, this experiment replicated the null findings of Experiment $1 \mathrm{~A}$. Significant effects were found for the reaction time measure; however, two factors argue against interpreting this outcome as evidence for time-of-semester effects. First, the observed effect was small: although the main effect of time of semester was significant, this main effect was qualified by the blocks $\times$ time interaction, for which estimated $\omega^{2}$ was .02 . Second, the pattern of results, as shown in Figure 2, does not correspond to an outcome that would be predicted on the basis of anticipated timeof-semester effects. End-of-semester subjects exhibited the slowest reaction times during the first block of trials but also showed a larger practice effect than did any other group. Thus, these subjects were actually faster overall than early-semester subjects (the difference was significant by $t$ test) and speeded their responses significantly with practice (simple effect of blocks was significant for the late-semester group). Given the absence of significant differences on the $d^{\prime}$ measure, indicating that all subjects responded accurately despite differences in the pattern of response times, no evidence was found for diminished performance by the late-semester group.

To extend the generality of this result, we examined time of semester effects using a more cognitively demanding task in Experiment 2.

\section{EXPERIMENT 2}

The subjects in Experiment 2 read a number of illustrated texts, using Just, Carpenter, and Wooley's (1982) moving-window procedure. Texts were selected from the stimuli used by Glenberg and Kruley (1992); each text described an object that should be unfamiliar to the average subject (e.g., the Sphagnum Sporophyte). After reading the text, the subjects answered a series of questions about it. Each text contained two anaphors-that is, noun phrases that referred to an object that had been mentioned earlier-requiring the reader to resolve the anaphor's referent (antecedent) in order to comprehend the sentence. These anaphors varied according to the distance in the text separating each anaphor from its antecedent; greater distances separating antecedent and anaphor have been found to challenge readers' comprehension abilities more severely (e.g., Clark \& Sengul, 1979). To answer the questions at the end of the text correctly, the subjects had to resolve the anaphor while reading. Note that the moving-window paradigm made it impossible to look back through the text to resolve the anaphor. Thus, the subjects were forced to rely on memory to make the appropriate connection.

If the results of Experiment 1 are limited to perceptual tasks, one might expect to see changes in subject performance in the more cognitively taxing task of Experiment 2. If, on the other hand, the results from Experiment 1 generalize to more cognitive tasks as well, one would expect no systematic changes in performance across the semester.

\section{Method}

Subjects. One hundred eighteen right-handed students in the University of Wisconsin-Madison subject pool participated in this experiment in exchange for extra credit. Approximately $\mathbf{4 0}$ subjects participated in each of three 2-week blocks (the same time periods used in Experiment 1B) in the Fall semester of 1993.

Design. The design was a mixed factorial; time of semester was the between factor with three levels (early, middle, and late), and anaphor distance was the within factor with two levels (near and far). The dependent measure was proportion correct on the comprehension questions.

Materials and Procedure. Texts and illustrations were displayed on Macintosh computers, and the subjects used the computer keyboard to respond. The texts for this experiment were taken from Glenberg and Kruley (1992). In outline, all subjects read 12 texts using the Just et al. (1982) moving-window paradigm. The illustrations that accompanied the texts were presented on the computer screen next to the text body. Each text contained two anaphors: one whose antecedent occurred in the sentence immediately preceding the anaphor sentence (near anaphor), and one whose antecedent occurred several sentences earlier in the text ( far anaphor).

After each text, the subjects answered three multiple-choice questions: one about the near anaphor, one about the far anaphor, and one filler question. Four possible answers were provided for each question.

\section{Results}

Figure 3 shows the mean levels of performance on the comprehension questions as a function of anaphor distance and time of semester; the overall mean was $78.53 \%$ correct. In the ANOVA, the distance $\times$ time interaction was nonsignificant $\left[F(2,115)=1.10, M S_{\mathrm{e}}=89.81\right.$, estimated $\left.\omega^{2}=.00\right]$. The main effect of distance was significant $\left[F(1,115)=29.46, M S_{\mathrm{e}}=89.81\right]$, and the main effect of time of semester approached but did not attain significance $\left[F(2,115)=2.86, M S_{\mathrm{e}}=393.84\right.$, estimated $\omega^{2}=$ $.03, p<.07]$. 


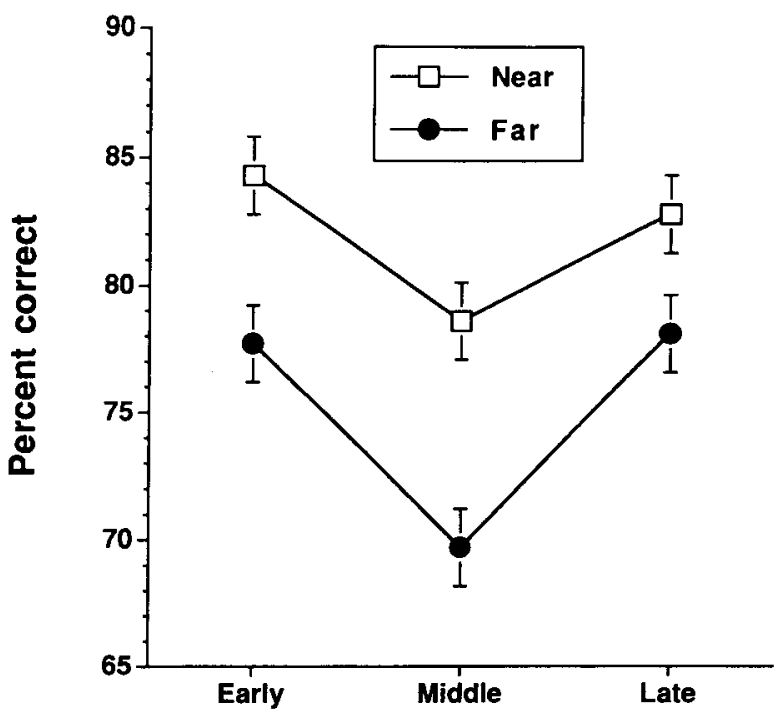

Time of semester

Figure 3. Mean percent correct on comprehension questions in Experiment 2, as a function of anaphor distance and time of semester. Error bars were computed as in Figures 1 and 2.

\section{Discussion}

The lowest level of overall comprehension occurred midsemester, with early and late subjects performing about equally well (the difference between these two groups was not significant by a $t$ test, the most powerful applicable method). Of greatest relevance to the question of differential subject performance during the semester is the absence of a significant distance $\times$ time interaction. The anaphor distance effect did not change detectably during the semester, and the simple effect of distance was significant at all three time periods. Thus, although performance fluctuated somewhat during the semester, the effect of theoretical interest to studies of text comprehension did not.

\section{GENERAL DISCUSSION}

Taking our results as a whole, we found no evidence to support the various hypotheses suggesting that late-semester subjects produce data that are somehow deficient in quality. At this point, a note about generalizability is in order. Because the signal detection task can reasonably be expected to be sensitive to changes in subjects' readiness to perform or amount of effort expended, our conclusion would appear applicable to general performance tasks requiring accurate classification and rapid responding. Additionally, this conclusion can be extended to other more cognitively demanding tasks, such as reading comprehension. Admittedly, there are many types of experimental tasks that we did not investigate; for example, our studies did not require subjects to interact extensively with the experimenter or to provide measures of affective response. We also grant that some studies cited earlier found changes in subject demographics during the semester. We do maintain, however, that the available evidence regarding changes in experimental performance supports the view that these changes are small or nonexistent.

Thus, our results should provide encouragement for researchers who use introductory psychology students as subjects. In sum, our conclusion is very similar to that stated 30 years ago by Underwood et al. (1964): "systematic shifts over a school term are likely to be less than might have been anticipated" (p. 225).

\section{REFERENCES}

Blatt, S. J., \& Quinlan, P. (1967). Punctual and procrastinating students: A study of temporal parameters. Journal of Consulting Psychology, 31, 169-174.

Clark, H. H., \& Sengul, C. J. (1979). In search of referents for nouns and pronouns. Memory \& Cognition, 7, 35-41.

COHEN, J. (1988). Statistical power analysis for the behavioral sciences (2nd ed.). Hillsdale, NJ: Erlbaum.

Evans, R., \& DonNerstein, E. (1974). Some implications for psychological research of early versus late term participation by college subjects. Journal of Research in Personality, 8, 102-109.

Glenberg, A. M., \& KRULEY, P. (1992). Pictures and anaphora: Evidence for independent processes. Memory \& Cognition, 20, 461-471.

Green, D. M., \& Swets, J. A. (1966). Signal detection theory and psychophysics. New York: Wiley.

Holmes, D. S. (1967). Amount of experience in experiments as a determinant of performance in later experiments. Journal of Personality \& Social Psychology, 7, 403-407.

Just, M., Carpenter, P., \& Wooley, J. (1982). Paradigms and processes in reading comprehension. Journal of Experimental Psychology: General, 111, 228-238.

KEPPEL, G. (1991). Design and analysis: A researcher's handbook (3rd ed.). Englewood Cliffs, NJ: Prentice-Hall.

Richter, D. O., Wilson, S. D., Milner, M., \& SENTER, R. J. (1981). Some differences among students volunteering as research subjects. Bulletin of the Psychonomic Society, 17, 261-263.

UNDERWOOD, B. J., SCHWENN, E., \& KePPEL, G. (1964). Verbal learning as related to point of time in the school term. Journal of Verbal Learning \& Verbal Behavior, 3, 222-225.

\section{NOTES}

1. The median was used to control for outliers. The analyses were also performed using mean reaction times (excluding reaction times more than 2.5 standard deviations from a subject's mean). The results of the analyses of the mean reaction times were the same as those of the median analyses.

2. The value of 0.27 was obtained by interpolation in Cohen's (1988) Table 8.3.20, with $u=12$ and $n=20$. Because $u=13$ in the actual analyses, this is a conservative estimate.

(Manuscript received September 2, 1993; revision accepted for publication December 28, 1993.) 